\title{
Risiko Pricktest
}

Pricktests sind schnell, einfach und kostengünstig durchzuführen und gelten als sicher. Vereinzelt kommen jedoch auch systemische Reaktionen bei Testung vor. Kinderärzte aus Schweden ermittelten die Häufigkeit von Nebenwirkungen und suchten nach potenziellen Risikofaktoren.

E ine Arbeitsgruppe aus dem schwedischen Hudiksvall wertete in einer prospektiven Studie die Daten von 5.908 Patienten aus, bei denen in elf pädiatrischen Kliniken und Praxen ein Pricktest vorgenommen worden war. Die Teilnehmer hatten ein Alter von höchstens 18 Jahren und die betreuenden Pflegekräfte waren angehalten, alle unerwarteten Ereignisse zu dokumentieren.

Insgesamt wurden 14 Nebenwirkungen verzeichnet: sieben systemische allergische Reaktionen, die medikamentös behandelt werden mussten, und sieben vasovagale Reaktionen, inklusive Synkopen. Das Risiko lag jeweils bei 0,12\%. $\mathrm{Zu}$ den Faktoren, die die Wahrschein- lichkeit für eine systemische Reaktionen erhöhten, gehörten ein junges Alter $(<1$ Jahr, relatives Risiko [RR] 6,28) und ein aktives Ekzem (RR 16,98). Das Risiko für vasovagale Reaktionen wurde durch weibliches Geschlecht (RR 7,32) und mehrere Pricktests bei einem Patienten ( $\mathrm{p}<0,05)$ erhöht.

Fazit: Systemische Reaktionen im Rahmen eines Pricktests sind zwar selten, aber möglich. Hierfür konnten Risikofaktoren ermittelt werden.

pe

Norrman G et al. Adverse reactions to skin prick testing in children - prevalence and possible risk factors. Pediatr Allergy Immunol 2009; 20: 273-8

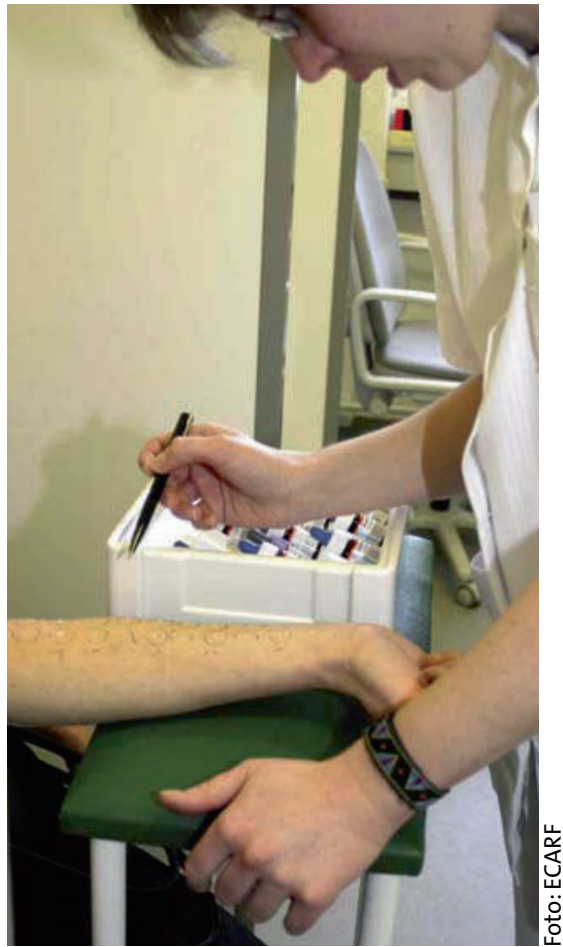

Der Pricktest gehört zum diagnostischen Standard-Repertoire in der Allergologie sehr selten kann er zu Allgemeinreaktionen führen.

\section{Kontaktallergie beginnt früh}

\section{Schon Kleinkinder unter fünf Jahren können gegen gängige Kontakt-} allergene sensibilisiert sein. Das zeigen die Ergebnisse einer niederländischen Studie.

P ädiatrische Dermatologen der Erasmus-Universität in Rotterdam analysierten prospektiv die PatchtestErgebnisse von Kindern mit einer therapierefraktären Dermatitis, die zur Abklärung einer vermuteten Kontaktallergie in ihre Klinik gekommen waren. Verwendet wurden die Standardreihen 1 und 2 des TRUE $^{\circledR}$-Epikutantests ergänzt um Tixokortol-17Pivalat, Budesonid und drei gängige Emollienzien. Ergänzende Tests wurden mit weiteren Allergenen bei gegebenem Verdacht vorgenommen.

Insgesamt 31 Jungen und 48 Mädchen wurden getestet. 40 von ihnen, und damit mehr als jedes zweite Kind mit Verdacht auf eine Kontaktallergie, reagierten tatsächlich mindestens ein- mal im Epikutantest. Von diesen Kindern litten 22 an einer atopischen Dermatitis, neun an einer Hand- oder Fußdermatitis und neun an anderen Hautbeschwerden. Wie bei den Erwachsenen war auch bei diesen Kindern Nickel das häufigste nachgewiesene Kontaktallergen, $43 \%$ reagierten im Test auf Nickelsulfat. Aber auch eine Vielzahl anderer bekannter Kontaktallergene wie beispilesweise Kaliumdichromat, Duftstoffmix oder Thiomersal lösten Reaktionen aus. Die Emollienzien riefen nur in Einzelfällen ein positives Testergebnis hervor. Bei sechs Kindern wies ein zusätzlicher Test auf die Schuhe als Auslöser einer Kontaktallergie hin, bei fünf auf Schienbeinschützer.
In der vorliegenden niederländischen Untersuchung waren fünf Kinder mit einem oder mehreren positiven Testergebnissen unter fünf Jahren alt. Auch aus der Literatur sind sehr frühe Fälle von Kontaktdermatitiden bekannt bis hin zu einem erst eine Woche alten Neugeborenen mit einer Kontaktallergie gegen ein Epoxidharz, das im Namensarmband der Klinik enthalten war.

Fazit: Kontaktallergien sind bei Kindern keineswegs eine Rarität und müssen bei Ekzemen, insbesondere bei atopischen Ekzemen, in Betracht gezogen werden. Zur Identifizeirung der Allergene sind Epikutantests notwendig. $f k$

de Waard-van der Spek FB et al. Patch tests in children with suspected allergic contact dermatitis: a prospective study and review of the literature. Dermatology 2009; 218: 119-25 